\title{
Performance Evaluation of the Optimal Hierarchy for Cellular Networks
}

\author{
So-Jeong Park ${ }^{1, *}$, Gyung-Leen Park ${ }^{1, * *}$, In-Hye Shin ${ }^{1}$, Junghoon Lee ${ }^{1}$, \\ Ho Young Kwak ${ }^{2}$, Do-Hyeon Kim², Sang Joon Lee ${ }^{2}$, and Min-Soo Kang ${ }^{3}$ \\ ${ }^{1}$ Department of Computer Science and Statistics, Cheju National Univ., Korea \\ \{joypark, glpark, ihshin76, jhlee\} @cheju.ac.kr \\ ${ }^{2}$ Faculty of Telecommunication and Computer Engineering, Cheju National Univ., Korea \\ \{kwak, kimah, sjlee\} @cheju.ac.kr \\ ${ }^{3}$ Department of Information and Communication Engineering, Hanyangcyber Univ., Korea \\ mskang@hycu.ac.kr
}

\begin{abstract}
Reducing the location update cost has been a critical research issue since the location update process requires heavy signaling traffics. One of the solutions is employing the hierarchical structure in cellular networks to reduce the location update cost. This paper is not only to measure the average location update cost in cellular networks using the hierarchical structure but also to determine the degree of the hierarchy which minimizes the location update cost. The paper proposes an advanced analytical model to obtain the optimal hierarchy in cellular networks, considering the update rates of the two registers, HLR and VLR, as well as the update delays for them. Also, the paper provides the threshold values as a guideline for the network administrator to design the optimal hierarchical structure in cellular networks.
\end{abstract}

\section{Introduction}

Many researchers have studied on diverse design problems of the location management schemes in cellular networks. The location management means the task finding the location of the mobile station (MS) in order to receive the seamless services properly while the MS moves [1-3]. The cellular networks has two types of databases, the home location register (HLR) and the visitor location register (VLR). The mobile switching center (MSC) associated with a specific VLR is in charge of several base station controllers (BSCs), lower control entities which in turn control several base stations (BSs). The MSCs are connected to the backbone wired network such as public switching telephone network (PSTN). The network coverage area is divided into smaller cell clusters called location areas (LAs). The VLR stores temporarily the service profile of the MS roaming in the corresponding LA while the HLR stores permanently the user profile and points to the VLR associated with the LA where the user is currently located.

* This research was supported by the MIC (Ministry of Information and Communication), Korea, under the ITRC support program supervised by the IITA (IITA-2006-C1090-06030040).

** The corresponding author. 
The total location update cost consists of the update cost of HLR and that of VLR. Since the HLR is connected to many VLRs, it has heavy signaling traffics [4-6]. The super location area (SLA) [7], a group of LAs, has been proposed for hierarchical networks to reduce the location update cost of the HLR. The paper develops an analytical model to obtain the optimal size of SLA which minimizes the total location update cost which includes both of the update rates of the HLR and VLR as well as the update delays for them. Also, the paper provides threshold values as a guideline that allows network administrators to design an optimal hierarchical structure for cellular networks depending on the given environments.

\section{The Location Management Using the Hierarchical Structure}

An example of the architecture of the scheme is depicted in Figure 1 [7].

Each SLA consists of 7 LAs and again each LA has 7 cells as shown in Figure 1. Each MSC/VLR pair covers an SLA. When an MS enters a new LA, the LA could belong to either a different SLA or the same SLA. If the LA belongs to a different SLA, both of the VLR and the HLR are updated to record the LA where the MS is located. On the other hand, if the new LA belongs to the same SLA, only the VLR is updated to record the new LA. The conventional scheme, which doesn't employ any hierarchy, should update not only the VLR but also the HLR whenever an MS enters a new LA regardless of any SLA. The previous research [7] could not answer the questions like "What is the optimal number of levels in the hierarchy when designing cellular networks?". The next section proposes an analytic model to answer the questions.

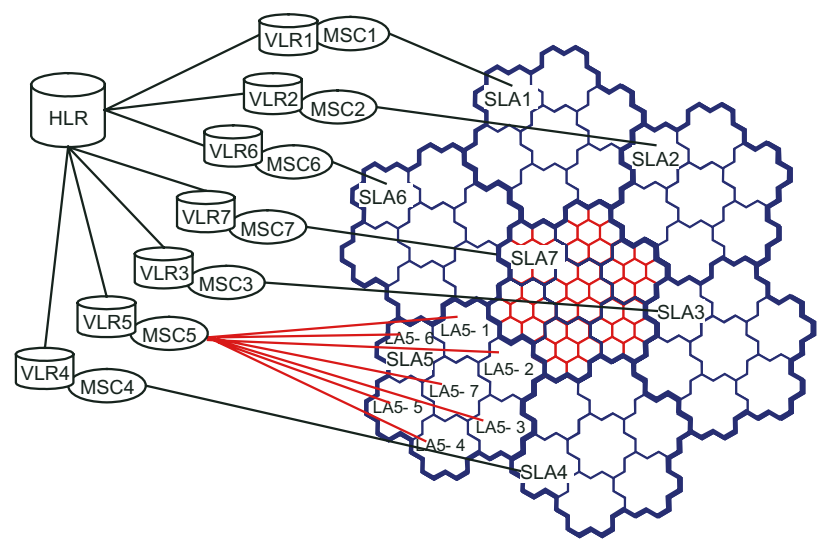

Fig. 1. The cellular architecture using hierarchical structure

\section{The Proposed Analytical Model}

This section evaluates the total update rate of the VLR and that of the HLR as well as the total update delay of the VLR and that of the HLR. 
The followings assumptions are made in the model.

1. The service area is divided into hexagonal cells of the equal size.

2. A mobile user moves independently to one of neighboring cells with the uniform distribution.

3. The dwell time in any cell for a mobile user is an exponentially distributed random variable with the average value, $\overline{T_{d}}$.

4. The update cost between a cell and a neighboring cell is 1 .

The notations used in the proposed model are depicted in Table 1.

Table 1. The Notations Used in the Analytic Model

\begin{tabular}{|c|l|}
\hline $\bar{K}$ & The average number of mobile users in a cell \\
\hline$K_{M S}$ & The total number of mobile users in the whole network \\
\hline$d$ & The size of an LA \\
\hline$\overline{T_{d}}$ & The average dwell time of a mobile user \\
\hline$N_{c}$ & The number of cells in an LA $: 3 d^{2}-3 d+1$ \\
\hline$N_{b c}$ & The number of boundary cells in an LA $: 6(d-1)$ \\
\hline$N_{S l a}$ & The number of LAs in an SLA $: 3 d_{s}^{2}-3 d_{s}+1$ \\
\hline$N_{S c}$ & The number of cells in an SLA : $\left(3 d^{2}-3 d+1\right)\left(3 d_{s}{ }^{2}-3 d_{s}+1\right)$ \\
\hline$N_{S b L A}$ & The number of boundary LAs in an SLA $: 6\left(d_{s}-1\right)$ \\
\hline$\overline{R_{L A}}$ & The average location update rate per mobile user in an LA \\
\hline$R_{V L R}$ & The total location update rate of VLR \\
\hline$\overline{R_{S L A}}$ & The average location update rate per mobile user in an SLA \\
\hline$R_{H L R}$ & The total location update rate of the HLR \\
\hline$d_{T}$ & The size of the whole cellular network \\
\hline$\left[d_{T}\right]$ & The greatest positive number not larger than $d_{T}$ \\
\hline$K_{S L A}$ & The number of SLAs in the whole network \\
\hline$K_{b S L A}$ & $\begin{array}{l}\text { The number of SLAs which does not form complete rings in the whole } \\
\text { network }\end{array}$ \\
\hline$l_{L A}$ & The distance between the centers of two neighboring LAs \\
\hline$r$ & The average distance from the center of an SLA to the corresponding ring \\
\hline$l_{S L A}$ & The distance between the centers of two neighboring SLAs \\
\hline$l_{S L A 1}$ & $\begin{array}{l}\text { The longest distance among the center of an SLA and the boundary cells } \\
\text { in the SLA }\end{array}$ \\
\hline$\overline{D_{V L R}}$ & The average location update delay of the VLR \\
\hline$\overline{D_{H L R}}$ & The average location update delay of the HLR \\
\hline$C$ & The total location update cost in whole network \\
\hline
\end{tabular}

In Figure 2, the LA consists of 19 cells. The boundary LAs of the SLA are classified into 6 vertex LAs and 12 side LAs. A mobile user may cross the border (a boundary cell) with probability of $1 / 6,2 / 6$, or $3 / 6$ according to the location of the cell. 


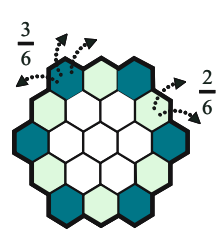

(a) An LA

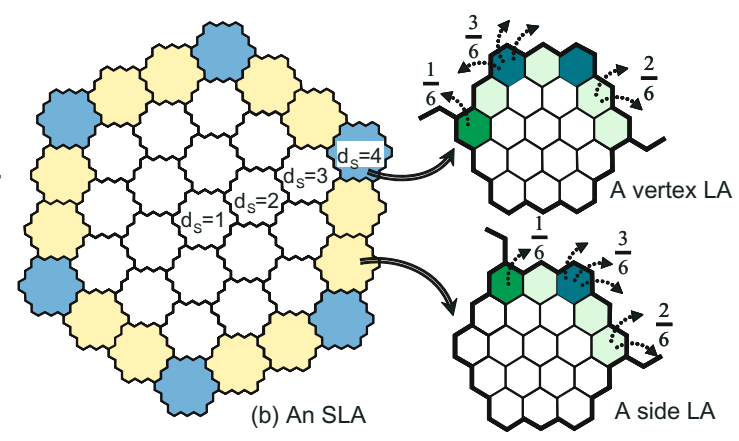

(b) An SLA

Fig. 2. (a) The Structure of an LA (b) The Structure of an SLA

The location update of the VLR occurs when a mobile user crosses the boundary cells of LA. Thus the total update rate of the VLR is obtained by Equation (2) which is derived from Equation (1) in [7], as shown in Figure 2-(a).

$$
\begin{gathered}
\overline{R_{L A}}=6 \times\left\{\frac{2}{6} \times\{6(d-2)\}+\frac{3}{6} \times 1\right\} \times \bar{K} \times \frac{1}{\overline{T_{d}}}=(2 d-1) \cdot \bar{K} \cdot \frac{1}{\overline{T_{d}}} \\
R_{V L R}=K_{S L A} \times\left(N_{S l a} \times \overline{R_{L A}}\right)=\frac{K_{M S}}{N_{S c} \times \bar{K}} \times\left(N_{S l a} \times(2 d-1) \times \bar{K} \times \frac{1}{\overline{T_{d}}}\right)=\frac{(2 d-1)}{\left(3 d^{2}-3 d+1\right)} \cdot K_{M S} \cdot \frac{1}{\overline{T_{d}}}
\end{gathered}
$$

When a mobile user moves into another SLA, the location update of the HLR happens in the boundary cells in the current SLA. Consequently, the total update rate of the HLR is obtained by Equation (4) using Equation (3) considering the vertex LAs and the side LAs, as shown in Figure 2-(b).

$$
\begin{gathered}
\overline{R_{S L A}}=6\left[\left(d_{s}-2\right)\left\{\frac{2}{6} \times 6(d-1)+1\right\}+\left\{\frac{3}{6} 6(d-1)+1\right\}\right] \times \bar{K} \times \frac{1}{\overline{T_{d}}}=\left(2 d_{s}-1\right)(2 d-2) \cdot \bar{K} \cdot \frac{1}{\overline{T_{d}}} \\
R_{H L R}=K_{S L A} \times \overline{R_{S L A}}=\frac{\left(2 d_{s}-1\right)(2 d-1)}{\left(3 d_{s}{ }^{2}-3 d_{s}+1\right)\left(3 d^{2}-3 d+1\right)} \cdot K_{M S} \cdot \frac{1}{\overline{T_{d}}}
\end{gathered}
$$

If a VLR is located at the center of an SLA, the update delay of the VLR can be regarded as the distance between the center of an SLA and the boundary cell of the SLA where the update of the VLR happens. In Figure 3, the shadowed parts, where the update of VLR occurs, are the boundary cells of LAs. The SLA forms three rings which connect the center of each LA. The rings are depicted as Ring1, Ring2, and Ring 3 in the figure. Then, the average update delay of the VLR is the average distance between the center of the SLA and the corresponding ring.

The average distance is obtained by calculating the radius of a circle. The distance, $l_{L A}$ is obtained by Equation (5) because $\triangle A B C$ is a regular triangle, where $\angle A C B=$ $60^{\circ}, \overline{A C}=\overline{A B}=\overline{B C}=(2 d-1)$, and $\overline{C D}=(d-1)$. 


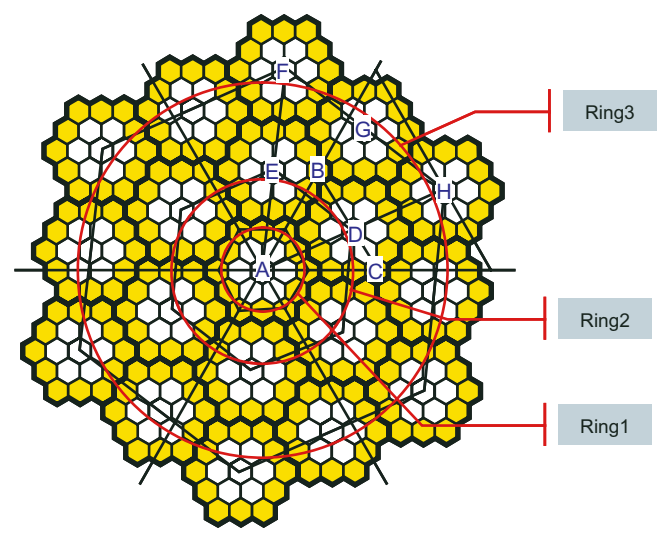

Fig. 3. The Architecture of an SLA with $d_{s}=3$

$$
l_{L A}=\overline{A D}=\sqrt{\overline{A C}^{2}+\overline{C D}^{2}-\overline{A C} \times \overline{C D}}=\sqrt{3 d^{2}-3 d+1}
$$

Therefore the average distance from the center of the SLA to the corresponding ring is obtained by Equation (6). The average update delay of the VLR, consequently, is obtained by Equation (7).

$$
\begin{gathered}
r=\sqrt{\frac{3 \sqrt{3}}{2 \pi}} \times \overline{F H}=\sqrt{\frac{3 \sqrt{3}}{2 \pi}} \times\left(d_{s}-1\right) \times l_{L A} \\
\overline{D_{V L R}}=\frac{1 \times \sqrt{\frac{3 \sqrt{3}}{2 \pi}} \cdot(d-1)+6 \sqrt{\frac{3 \sqrt{3}}{2 \pi}} \cdot l_{L A}+12 \sqrt{\frac{3 \sqrt{3}}{2 \pi}} \cdot 2 \cdot l_{L A}+\cdots+\frac{6\left(d_{s}-1\right) \sqrt{\frac{3 \sqrt{3}}{2 \pi}} \cdot\left(d_{s}-1\right) \cdot l_{L A}}{N_{S l a}}}{N_{S l a}} \\
=\frac{\sqrt{\frac{3 \sqrt{3}}{2 \pi}} \cdot(d-1)+\sqrt{\frac{3 \sqrt{3}}{2 \pi}} \cdot \sqrt{3 d^{2}-3 d+1} \cdot\left(2 d_{s}{ }^{3}-3 d_{s}{ }^{2}+d_{s}\right)}{3 d_{s}{ }^{2}-3 d_{s}+1}
\end{gathered}
$$

Assume that HLR is centrally located in the network because the HLR is logically only one in the whole network.

In Figure $4, \triangle A B C$ is a regular triangle and $\angle A C B$ is $60^{\circ} . \overline{A C}$ and $\overline{C D}$ are obtained by Equation (8) and (9), respectively. Consequently, the distance between SLA1 and SLA2, $l_{S L A}$, is obtained by Equation (10).

$$
\begin{aligned}
\overline{A C}=\overline{A E}+(\overline{F G}+\overline{H I})-(\overline{J K}+\overline{L M})+(\overline{N O}+\overline{P Q})+\overline{R D} \\
=(d-1)+\left(d_{s}-1\right)(2 d-1)-\left(d_{s}-1\right)(d-1)+\left(d_{s}-1\right)(2 d-1)+d=3 d d_{s}-d-d_{s} \\
\overline{C D}=(\overline{E F}+\overline{G H}+\overline{I J})+(\overline{K L}+\overline{M N})+(\overline{O P}+\overline{Q R}) \\
=d_{s}(d-1)+\left(d_{s}-1\right)(d-1)=3 d d_{s}-2 d-2 d_{s}+1
\end{aligned}
$$




$$
l_{S L A}=\overline{A D}=\sqrt{\overline{A C}^{2}+\overline{C D}^{2}-\overline{A C} \times \overline{C D}}=\sqrt{9 d^{2} d_{s}^{2}-9 d^{2} d_{s}-9 d d_{s}^{2}+9 d d_{s}+3 d^{2}+3 d_{s}^{2}-3 d-3 d_{s}+1}
$$

The size of the whole cellular networks is obtained by Equation (11) because the SLAs are arranged in rings.

$$
d_{H}=\frac{1}{2}+\sqrt{\frac{1}{4}-\frac{1}{3}\left(1-K_{S L A}\right)}
$$

Outer cells in the network cannot complete the ring for the SLA configuration. The largest size of a complete ring is the greatest positive number not larger than $d_{H}$. The number of SLAs which do not complete their outer rings is in Equation (12).

$$
K_{b S L A}=K_{S L A}-\left(3\left[d_{H}\right]^{2}-3\left[d_{H}\right]+1\right)
$$

As shown in Figure 4, if $d_{H}=1$ then the average updating delay of HLR is the average distance between the center and the boundary cell in the central SLA. $\triangle A S T$ is a regular triangle and $\angle A T I$ is $60^{\circ} . \overline{A T}$ and $\overline{I T}$ are obtained in Equation (13) and (14), respectively. Therefore the longest distance, $l_{S L A 1}$, is obtained by Equation (15).

$$
\begin{gathered}
\overline{A T}=\overline{A E}+(\overline{F G}+\overline{H I})=(d-1)+\left(d_{s}-1\right)(2 d-1)=2 d d_{s}-d-d_{s} \\
\overline{I T}=(\overline{E F}+\overline{G H})=\left(d_{s}-1\right)(d-1)=d d_{s}-d-d_{s}+1 \\
l_{S L A 1}=\overline{A I}=\sqrt{\overline{A T}^{2}+\overline{I T}^{2}-\overline{A T} \times \overline{I T}}=\sqrt{3 d^{2} d_{s}{ }^{2}-3 d^{2} d_{s}-3 d d_{s}{ }^{2}+2 d d_{s}+d^{2}+d_{s}{ }^{2}-d-d_{s}+1}
\end{gathered}
$$

Consequently, the average update delay of HLR is obtained by Equation (16).

$$
\begin{aligned}
\overline{D_{H L R}}= & \frac{\sqrt{\frac{3 \sqrt{3}}{2 \pi}} \times l_{S L A 1}+6 \sqrt{\frac{3 \sqrt{3}}{2 \pi}} \times l_{S L A}+12 \sqrt{\frac{3 \sqrt{3}}{2 \pi}} \times 2 \times l_{S L A}}{K_{S L A}}+\cdots \\
& +\frac{6\left(\left[d_{H}\right]-1\right) \sqrt{\frac{3 \sqrt{3}}{2 \pi}} \times\left(\left[d_{H}\right]-1\right) \times l_{S L A}+K_{b S L A} \times \sqrt{\frac{3 \sqrt{3}}{2 \pi}} \times\left[d_{H}\right] \times l_{S L A}}{K_{S L A}} \\
& =\frac{\sqrt{\frac{3 \sqrt{3}}{2 \pi}} \times l_{S L A 1}+\sqrt{\frac{3 \sqrt{3}}{2 \pi}} \times l_{S L A} \times\left(2\left[d_{H}\right]^{3}-3\left[d_{H}\right]^{2}+\left[d_{H}\right]\right)}{K_{S L A}}+\frac{K_{b S L A} \times \sqrt{\frac{3 \sqrt{3}}{2 \pi}} \times\left[d_{H}\right] \times l_{S L A}}{K_{S L A}}
\end{aligned}
$$

Finally, the total location update cost is obtained by Equation (17).

$$
C=R_{V L R} \times \overline{D_{V L R}}+R_{H L R} \times \overline{D_{H L R}}
$$

The more details of the derivations are omitted due to the space limit. Interested readers may refer to [8]. 


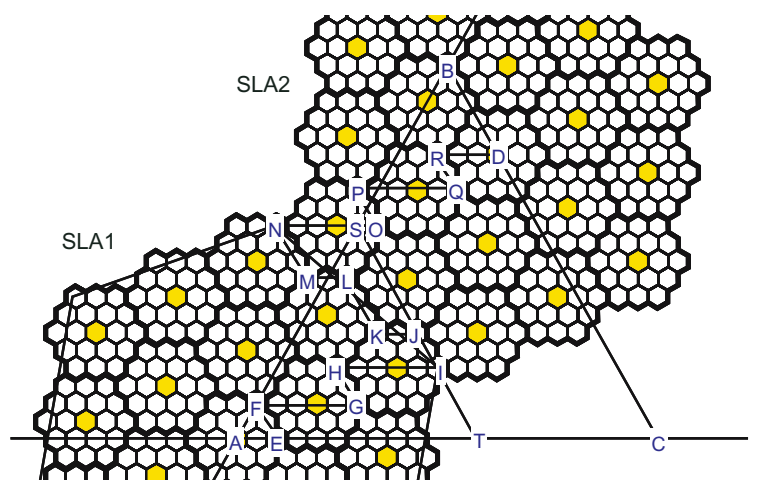

Fig. 4. The central SLA and a neighboring SLA

\section{The Results of Performance Evaluation}

This section shows the results of performance evaluation in terms of the total location update cost calculated using the model developed in the previous section.

Figure 5-(a) shows the total update cost according to the dwelling time while Figure 5-(b) does according to the size of an LA. The optimal size of an SLA is 7 irrespective of dwelling time as shown in Figure 5-(a). Figure 5-(b) shows that the larger the LA size is, the smaller the optimal size of an SLA become. The optimal size of an SLA is 14, 7, and 5 when the LA size=1, 3, and, 5, respectively.
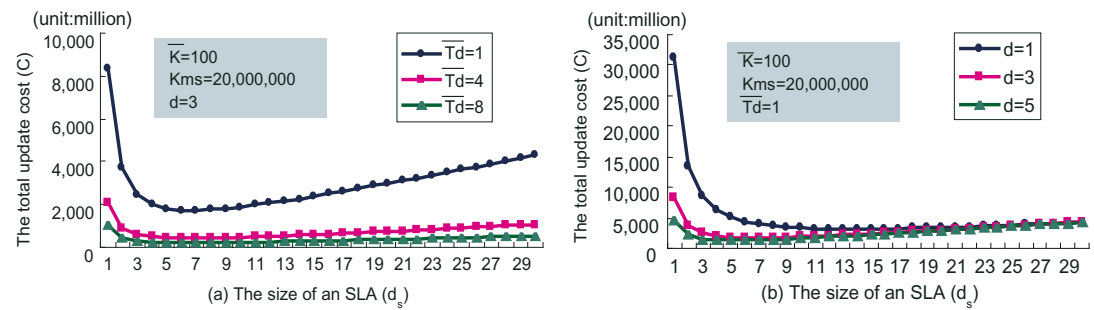

Fig. 5. The total update cost according to the dwelling time (a) and the LA size (b)
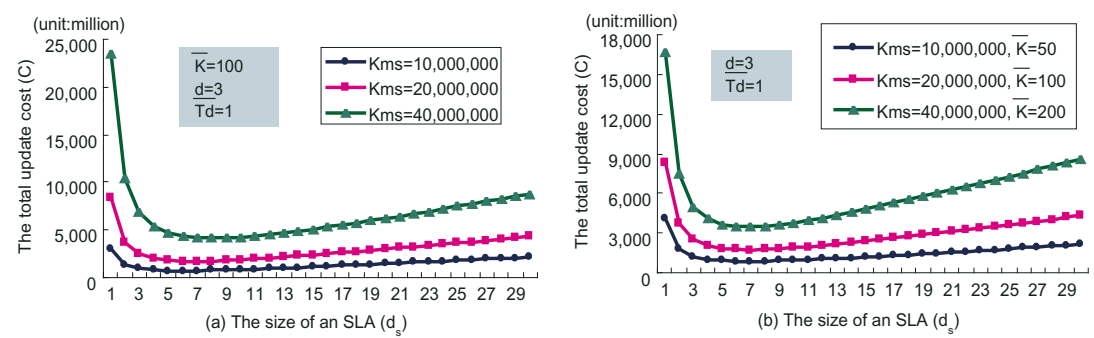

Fig. 6. The total update cost according to $K_{M S}$ (a) and $K_{M S}$ with same number of cells (b) 
Figure 6-(a) shows that the larger the number of the total mobile users is, the larger the optimal size of an SLA is. The optimal size of an SLA is 6, 7, and 8 when $K_{M S}=$ 10, 20, and 40 billion, respectively. Figure 6-(b) shows a change of the total update cost according to the total mobile users in the same number of cells $\left(K_{M S}\right.$ $/ \bar{K}=200,000$ ) in the network. The optimal size is constantly 7 in all cases.

\section{Conclusion}

The paper proposed an analytical model to obtain the optimal size of the SLA in cellular networks, considering the update rates of the HLR and that of the VLR as well as the update delays for them. The result obtained from the model shows that the number of cells in the whole cellular network affects sensitively the optimal size of SLA. Also, the model provides the threshold values as a guideline when network administrators aim to design the optimal hierarchical structure for cellular networks.

\section{References}

1. Kyamakya, K., Jobmann, K.: Location Management in Cellular Networks: Classification of the Most Important Paradigms, Realistic Simulation Framework, and Relative Performance Analysis. The IEEE Vehicular Technology Conference, Vol. 54. (2005) 687-708

2. Ali, S.Z.: Location Management in Cellular Mobile Radio Networks. IEEE International Symposium on PIMRC, Vol. 2. (2002) 745-749

3. Assouma, A.D., Beaubrun, R., Pierre, S.: A Location Management Scheme for Heterogeneous Wireless Networks. IEEE International Conference on Wireless and Mobile Computing, Networking And Communication, Vol. 2. (2005) 51-56

4. Kim, S., Smari, W.W.: Reducing Location Management Costs in Hierarchical-based Model of Mobile and Wireless Computing Systems. IEEE International Conference on Information Reuse and Integration, (2003) 428-435

5. Morris, D., Aghvami, A.H.: A Novel Location Management Scheme for Cellular Overlay Networks. IEEE Transaction on Broadcasting, Vol. 52. (2006) 108-115

6. Fan, G., Zhang, J.: A Multi-layer Location Management Scheme that Bridges the Best Static Scheme and the Best Dynamic Scheme. IEEE International Conference on Mobile Data Management, (2004) 757-760

7. In-Hye Shin, Gyung-Leen Park.: On Employing Hierarchical Structure in PCS Networks. ICCSA (2) (2003) 155-162

8. So-Jeong Park, Gyung-Leen Park.: An Analytical Model for Performance Evaluation of Hierarchical Structure in Cellular Networks. Tech. Report \#06-122, Cheju National University, Korea. 